Case Series

\title{
Case Series on Chronic Whiplash Related Neck Pain Treated with Intraarticular Zygapophysial Joint Regeneration Injection Therapy
}

R. Allen Hooper ${ }^{1}$, MD, J Bevan Frizzell², MD, and Peter Faris ${ }^{3}$, PhD

From: ${ }^{1}$ Advanced Spinal Care Centre, Calgary, Alberta, Canada, and 1,2,3 University of Calgary, Alberta, Canada

${ }^{1} \mathrm{Dr}$. Hooper is Clinical Assistant Professor, University of Calgary, and Director Clinical Services, Advanced Spinal Care Centre, Calgary, Alberta,

Canada. ${ }^{2}$ Dr. Frizzell is Section Head, Musculoskeletal Radiology, Foothills Medical Centre, Managing Partner, EFW Radiology and Clinical Assistant Professor, University of Calgary, Alberta, Canada. ${ }^{3}$ Dr. Faris

is Senior Biostatistician, Centre for Advancement of Health, Calgary, Alberta, Canada. Address Correspondence: R. Allen Hooper MD Clinical Assistant Professor, University of Calgary

Director Clinical Services, Advanced Spinal Care Centre G100A 2210 - 2nd Street SW Calgary, Alberta

Canada

$\mathrm{T}_{2} \mathrm{~S}_{3} \mathrm{C}_{3}$

E-mail: ahooper@ucalgary.ca Disclaimer: The authors provided funding for this study.

Conflict of interest: None Manuscript received: 10/26/2006 Revisions accepted: 1/17/2007 Accepted for Publication: 2/8/2007

Free Full manuscript: www.painphysicianjournal.com
Background: Although in clinical use, there is only 1 published case report on the efficacy of intraarticular regeneration injection therapy (RIT) (a.k.a. prolotheraphy). This report supports a rationale for future clinical trials of this technique.

Objective: To assess the efficacy of intraarticular zygapophysial joint RIT in patients with chronic whiplash related neck pain that failed other conservative and interventional procedures. Patients were treated with intraarticular RIT and reassessed over 1 year.

Design: Retrospective case review of prospective data.

Materials and Methods: Eighteen consecutive patients were treated with intraarticular prolotherapy by placing $0.5-1 \mathrm{~mL}$ of $20 \%$ dextrose solution into each zygapophysial joint, after confirmation of intraarticular location with radiographic contrast, using 25-gauge spinal needles and fluoroscopic guidance. Solution was prepared by diluting D50W with $1 \%$ lidocaine.

Results: Fifteen patients completed treatment. Three patients had bilateral treatment, leaving 18 sides for analysis. Mean Neck Disability Index (NDI) pre-treatment was 24.71 and decreased post-treatment to 14.21 (2 months), 13.45 (6 months), 10.94 (12 months). Average change NDI=13.77 ( $p<0.0001)$ baseline versus 12 months. Symptoms for 14 patients were from motor vehicle accident, of which 13 were in litigation. Patients attending physiotherapy over the course of treatment had better outcomes than those without physiotherapy. Women needed more injections (5.4) than men (3.2) $p=0.0003$.

Conclusion: Intraarticular RIT improved pain and function in this case series. The procedure appears safe, more effective than periarticular RIT, and lasted as long, or longer, than those patients with previous radiofrequency neurotomy. Concurrent physiotherapy helped reduce post-procedure neck stiffness. Future trials should consider gender when deciding how many treatments to administer. Litigation was not a barrier to recovery.

Key words: regenerative injection therapy, RIT, prolotherapy, zygapophysial joint, motor vehicle accident, whiplash

Pain Physician 2007; 10:313-318 


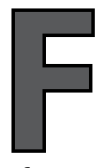

ollowing whiplash, injury to the zygapophysial capsule orcartilage(1)maybe a factor in ongoing zygapophysial joint pain. The prevalence of zygapophysial joint pain following whiplash injury is $60 \%$ (2). Radiofrequency (RF) neurotomy has been shown to provide up to a year of relief for zygapophysial joint pain (3). RF neurotomy is an effective pain management technique in appropriately selected patients, but does not necessarily address the underlying pathology. Intraarticular injection of dextrose and lidocaine (regenerative injection therapy - RIT, a.k.a. prolotherapy) may strengthen the zygapophysial joint capsule (4-6) and potentially have a beneficial effect on the cartilage $(7,8)$.

RIT has been used since 1935 to treat chronic pain conditions for spine, peripheral joints, and headache (9). Previous RIT studies for neck pain reported retrospectively on patient's impression of change for pain and function (10-12). Cervical periarticular RIT patients were less likely to report improvement in pain and ability to work compared to thoracic and lumbar spine patients. Better treatment techniques for cervical spine injuries were recommended (12). An intraarticular approach may allow better access to the anterior capsule not treated with a periarticular technique as well as having a potential effect on the cartilage.

In clinical use $(13,14)$, there is only 1 published report on cervical intraarticular RIT (15). The first patient treated in this current case series was previously presented as a case report with 3-year follow-up using the neck disability index (NDI) prospectively (15). The use of validated condition-specific disability questionnaires would help compare future studies.

The NDI is a 10-item scale that assesses pain and function. Each item is rated on a 6-point scale from 0 to 5 . The total score ranges from 0 to 50 , with lower scores indicating less pain and better function. It is the most frequently cited condition-specific self report measure for neck pain and research has proven it to be reliable and valid. The minimal detectable change and minimal clinically important change has been calculated to be 5 points (16).

This report documents the results on a case series of patients treated with intraarticular RIT. Our study helps to isolate RIT as an independent variable in the treatment of chronic spine pain. Manual physiotherapy with joint manipulation, dry needling of trigger points, and exercise were constants before and during the study. Further improvement was noted after adding intraarticular RIT.

\section{Methods}

Data collected for this study was part of a quality assurance program at a private outpatient clinic in Canada. Patients did sign consent prior to treatment. Although IRB approval was not sought, patient care meets the Declaration of Helsinki guidelines, in particular section 32 pertaining to new therapeutic measures and recording new information. Data collection began with the first patient treated with intraarticular RIT and continued consecutively until the government funded provincial health care system discontinued coverage for this treatment.

Eighteen selected patients had failed other conservative and interventional procedures such as physiotherapy, massage, acupuncture, and periarticular RIT. Six of the patients had previous RF neurotomy of the intraarticularly treated joints that provided temporary relief for 6 to 12 months.

Patients selected for intraarticular RIT were identified as having ligament laxity of the zygapophysial joint. Experienced physiotherapists performed manual assessment of the cervical spine. Shear stress testing of the cervical spine was performed by stabilizing the inferior segment laterally by blocking lateral motion at the articular mass, then applying lateral shear force from the opposite side to the articular mass of the superior segment. Antero-posterior stress testing of the cervical spine was performed by stabilizing the inferior bone anteriorly with both thumbs by sliding them in laterally underneath the prevertebral musculature, then applying anterior stress to the superior bone by engaging the neural arch posteriorly with the index fingers and providing an anterior strain to the articulation. In both tests, end-feel and amplitude of displacement were evaluated.

All patients underwent a single diagnostic zygapophysial joint injection with anesthetic to confirm that the lax joints were painful. The 6 patients with previous RF neurotomy to the lax levels also had a medial branch block with anesthetics of different duration to confirm the painful levels.

Joints deemed to be lax and painful were treated with intraarticular RIT by placing $0.5-1 \mathrm{~mL}$ of $20 \%$ dextrose solution into each zygapophysial joint, after confirmation of intraarticular location with radiographic contrast, using 25-gauge spinal needles and fluoroscopic guidance. The dextrose solution was prepared by diluting D50W with $1 \%$ lidocaine. Fluoroscopy of the subsequent joints injected revealed 
only infrequent leakage of solution from previously injected joints.

Prior to treatment, the patients received manual physiotherapy (including joint manipulation, dry needling of trigger points, and exercise), which was continued through the course of treatment. At the start of treatment, patients completed a neck disability index questionnaire. Injections were repeated every 1 to 2 months if manual physiotherapy identified ongoing laxity. The follow-up neck disability index questionnaires were mailed to patients 2,6 , and 12 months after their last treatment.

We used boxplots to examine the distribution of NDI scores at pre-test and at 2, 6, and 12 months. If distributions were reasonably symmetrical, we summarized these distributions using means and $95 \%$ confidence intervals. We used t-tests and $95 \%$ confidence intervals to evaluate change in NDI scores over time. Prior to conducting t-tests, we examined the distribution of change scores to assure that the distributions were approximately normal. We used all available data for each of the pair-wise t-tests. Because these tests were performed using sides rather than patients, the assumption of independence of observations was violated for 3 patients receiving treatments on 2 sides. We used linear mixed-effects models to assess the impact of these violations (17).

\section{Results}

Eighteen consecutive patients were selected for treatment. Two patients dropped out due to increasing stiffness after being unable to continue physiotherapy (private motor vehicle accident insurance benefits discontinued). One patient dropped out after becoming pregnant. Mean age of the 15 patients completing treatment was 36.07 (std 5.37) years, 11 of which were female. These patients had all received previous physiotherapy, and the majority other conservative or interventional care (massage $n=13$, chiropractic $n=11$, acupuncture $n=9$, periarticular RIT $n=10$, RF neurotomy $n=6$ ).

Symptoms for 14 patients were caused by motor vehicle accident (MVA), 13 of which were in litigation at the start of treatment. The 1 case not in litigation was hit by an uninsured driver out of country. Mean duration of symptoms for MVA cases was 48.88 (std 17.07) months. Duration of symptoms for the nonMVA case was 252 months.

Three of the 15 patients had bilateral treatment, leaving 18 sides for analysis. Each side was treated separately. One patient had significantly different results side to side (NDI change 3 on the left, 17 on the right). The other 2 patients with bilateral treatment reported identical changes for each side. Of the 15 patients with pre-treatment NDI scores, 2 patients (with 4 sides) were missing observations at 2 months, 6 patients (with 7 sides) were missing observations at 6 months, and 1 patient (1 side) was missing an observation at 12 months (Table 1 ).

Levels treated included C2-7 vertebral segments. Single level treated $=2$ cases, 2 levels treated $=9$ cases, 3 levels treated $=5$ cases, and 4 levels treated $=2$ cases. Total treatments per level were $C 2 / 3=10, C 3 / 4=13$, $C 4 / 5=12, C 5 / 6=7, C 6 / 7=1$. Mean number of treatments for males was 3.2 (std 0.45) and females 5.42 (std 1.44) $\mathrm{p}=0.0003$.

Four patients off work were able to return to regular duties. Two patients off work were able to return to modified duties. The remaining 9 patients continued working through the course of treatment. Patients generally found the injections uncomfortable, and reported a few days of stiffness afterward. No complications such as nerve injury or infection were encountered with the treatments administered in this case series.

Neck Disability Index scores with $95 \%$ confidence intervals at each time point are presented in Table 2. There is statistical evidence of improved NDI scores at each point in time. Linear mixed-effects models were used to assess the legitimacy of treating sides as independent observations when using t-tests. The results of these models were in agreement with the t-tests; treating sides as independent observations had minimal impact on the tests of significance.

\section{Discussion}

Intraarticular RIT improved pain and function in this case series. The 1-year relief observed in this series may extend longer. In our first case, NDI improved from 9 at 1 year to 2 at 3 years (15). The procedure appears safe, more effective than extraarticular prolotherapy, and may address the underlying pathology rather than just treat the symptoms as with RF neurotomy. Concurrent physiotherapy helped reduce post-procedure neck stiffness. Future studies should consider gender when determining how many injections to do. Litigation does not appear to be a barrier to recovery.

Poor responders to intraarticular RIT include those who were unable to continue physiotherapy ( 2 dropouts, 1 change in NDI=0, 1 change in NDI=5). The non- 
Table 1. Pre and post-treatment Neck Disability Index scores with levels treated

\begin{tabular}{|c|c|c|c|c|c|}
\hline Patient & Pre NDI & NDI 2 months & NDI 6 months & NDI 12 months & Levels treated \\
\hline 1 & 24 & 9 & missing & 9 & Right C2-6 \\
\hline 2 & 28 & 1 & missing & 11 & Left C2-4 \\
\hline 3 & 27 & 28 & 26 & 22 & Right C2-4 \\
\hline 4 & 10 & 4 & 4 & 4 & Right C2-4 \\
\hline 5 & 27 & 13 & missing & 5 & Left C2-4 \\
\hline 6 & 27 & 20 & 21 & 21 & Right C4-7 \\
\hline 7 & 27 & 10 & 9 & 4 & Left C3-5 \\
\hline 8 & 27 & 27 & missing & missing & Right C4-6 \\
\hline 9 & 21 & 10 & 13 & 8 & Right C2-6 \\
\hline 10 & 32 & 21 & 7 & 3 & Right C2-4 \\
\hline 11 left & 32 & 20 & missing & 20 & Left C4-5 \\
\hline 11 right & 32 & 20 & missing & 20 & Right C4-5 \\
\hline 12 left & 24 & missing & 15 & 21 & Left C2-5 \\
\hline 12 right & 24 & missing & 17 & 7 & Right C2-5 \\
\hline 13 left & 18 & missing & 14 & 8 & Left C3-6 \\
\hline 13 right & 18 & missing & 14 & 8 & Right C3-6 \\
\hline 14 & 17 & 9 & 8 & 10 & Right C4-6 \\
\hline 15 & 15 & 7 & missing & 5 & Left C2-4 \\
\hline
\end{tabular}

Table 2. Summary of pre and post-treatment Neck Disability Index scores.

\begin{tabular}{|l|l|l|}
\hline & Mean NDI score $\mathbf{x} / 50 \mathbf{( 9 5 \%}$ CI) & P-value \\
\hline Pre-treatment & $24.71(20.85,28.57)$ & \\
\hline 2 months & $14.21(9.35,19.08)$ & 0.0001 \\
\hline 6 months & $13.45(9.15,17.76)$ & 0.0017 \\
\hline 12 months & $10.94(7.39,14.50)$ & $<0.0001$ \\
\hline
\end{tabular}

CI - confidence interval

MVA patient (change in $\mathrm{NDI}=6$ ) started with a lower NDI score $=10$ and had a longer duration of symptoms. One patient had a change of $\mathrm{NDI}=3$ on one side and 17 on the other. We are clinically unable to account for this difference. The patient continues to require yearly RF neurotomy to control pain on the less responsive side. The remaining low responder's (change $\mathrm{NDI}=6$ ) continued to have shoulder pain and functional limitations with the shoulder. There were no distinguishing features between the remaining responders.

Litigation is not a factor in predicting disability in patients with chronic pain (18) or brain injury (19). However, research on patients with neck pain after whiplash demonstrates retaining a lawyer is an independent predictor of poor outcome (20). This may not be the case in subgroups where specific diagnosis and treatment is available. RF neurotomy for neck pain patients in litigation has been shown to be beneficial (21). Litigation was not a barrier to recovery in this series.

Previous retrospective data from this practice (12) indicated a gender difference in the number of treatments required which was shown in this series as well. Future research should consider this.

Several mechanisms of action for RIT have been proposed including, neuromodulation, regression of 
neovasculogenesis, release of adhesions, and stimulation of the inflammatory/proliferative/regenerative/reparative cascade mediated by cytokines and numerous growth factors (9). An intraarticular approach allows better access to the anterior capsule and cartilage that would allow these mechanisms to work on more structures than a periarticular approach.

\section{STUdY Limitations}

The small number of patients and lack of a control group limit this study, and we cannot be certain that the differences observed can be attributed solely to the effects of the prolotherapy. In addition, we opted to use data from all treated sides, and 3 of the 15 patients contributed data from both sides. Using linear mixed-effects models, we determined that any dependencies among observations had minimal impact on our results. However, more observations would be re- quired to fully investigate the statistical implications of using 2 sides from some patients.

Manual physiotherapy can be as accurate as radiologically controlled diagnostic blocks at determining the painful zygapophysial joint levels (22). The use of stress testing to diagnose ligament laxity however is limited by poor inter-rater reliability. A more objective assessment for ligament laxity may prove to be overpressure flexion views (23). Laxity may not necessarily be an important selection criterion. Painful zygapophysial joints identified with diagnostic blocks may respond equally well.

\section{Conclusions}

Following RIT, we observed clinical improvements in patients with chronic pain following whiplash. Despite the limitations of our study, the data provide some insights for discussion and provide a justification for future research.

\section{References}

1. Stemper BD, Yoganandan N, Pintar FA. Gender and region-dependent local facet joint kinematics in rear impact. Spine 2004;16:1764-1771.

2. Lord SM, Barnsley L, Wallis BJ, Bogduk N. Chronic cervical zygapophysial joint pain after whiplash. Spine 1996;21:1737-1745.

3. Lord SM, Barnsley L, Wallis BJ, McDonald GJ, Bogduk N. Percutaneous radiofrequency neurotomy for chronic cervical zygapophyseal-joint pain. $N$ Engl J Med 1996;335:1721-1726.

4. Klein RG, Dorman TA, Johnson CE. Proliferant injections for low back pain: histologic changes of injected ligaments and objective measurements of lumbar spine mobility before and after treatment. Journal of Neurological and Orthopaedic Medicine and Surgery 1989;10:141-144.

5. Lui YK, Tipton CM, Matthes RD, Bedford JA, Maynard JA, Walmer HC. An in-situ study of the influence of a sclerosing solution in rabbit medial collateral ligaments and its junction strength. Connective Tissue Research 1983;11:95102.

6. Maynard JA, Pedrini VA, Pedrini-Mille A, Romanus B, Ohlerking F. Morphological and biochemical effects of sodium morrhuate on tendons. Journal of Orthopaedic Research 1985;3:236-248.
7. Reeves KD. Randomized prospective double-blind placebo controlled study of dextrose prolotherapy for knee osteoarthritis with or without acl laxity. Alternative Therapies 2000a;6:68-80.

8. Reeves KD, Khatab H. Randomized, prospective, placebo-controlled doubleblind study of dextrose prolotherapy for osteoarthritic thumb and finger (dip, pip, and trapeziometacarpal) joints: evidence of clinical efficacy. The Journal of Alternative and Complementary Medicine 200ob;6:311-320.

9. Linetsky FS, Derby R, Miguel R, Saberski L, Stanton-Hicks M. Pain management with regenerative injection therapy. In Weiner's Pain Management: $A$ Practical Guide (7th Edition) 2006 Chapter 62.

10. Dorman T. Whiplash Injuries: treatment with prolotherapy and a new hypothesis. J Ortho Med 1999;21:13-21.

11. Hackett GS, Huang TC, Raftery A. Prolotherapy for headache: pain in the head and neck, and neuritis. Headache 1962;2:21-28.

12. Hooper RA, Ding M. Retrospective case series on patients with chronic spinal pain treated with dextrose prolotherapy. The Journal of Alternative and Complimentary Medicine 2004; 10:670-74.

13. Stanton-Hicks M. Cervicocranial syn- drome: Treatment of atlanto-occipital and atlanto-axial joint pain with phenol/glycerin injections. Presented at 2oth American Association of Orthopedic Medicine Annual conference and scientific seminar; A Common Sense Approach to Hidden Pain Generators. Orlando Fl 2003.

14. O’Neill C. Intra-articular dextrose/glucosamine injections for cervical facet syndrome, atlanto-occipital and atlanto-axial joint pain, combined ISIS AAOM approach. Presented at 2oth American Association of Orthopedic Medicine Annual conference and scientific seminar; A Common Sense Approach to Hidden Pain Generators. Orlando Fl 2003.

15. Hooper RA, Sherman ST, Frizzell JB. Case report of whiplash related chronic neck pain treated with intraarticular prolotherapy. Journal of Whiplash Related Disorders. 2005;4:23-27.

16. Stratford PW, Riddle DL, Binkley JM, Spadoni G, Westaway MD, Padfield B. Using the neck disability index to make decisions concerning individual patients. Physiotherapy Canada 1999;spring:107-119.

17. Pinheiro JC, Bates DM. Mixed effects models in $S$ and S-PLUS. New York: Springer. 2000.

18. Solomon P, Tunks E. The role of litiga- 
tion in predicting outcomes in chronic pain patients. Clin J Pain 1991;7:300304.

19. Wood RL, Rutterford NA. The effect of litigation on long term cognitive and psychosocial outcome after severe brain injury. Arch Clin Neuropsychology 2006;21:239-246.
20. Bogduk N. Point of view. Spine 2006;31: E766.

21. Sapir DA, Gorup JM. Radiofrequency medial branch neurotomy in litigant and nonlitigant patients with cervical whiplash. Spine 2001;26:E268-E273.

22. Jull G, Bogduk N, Marsland A. The accuracy of manual diagnosis for cervical zygapophysial joint pain syndromes. Med J Australia 1988;148:233-236

23. Centeno CJ, Elliot J, Elkins WL, Freeman M. Fluoroscopically guided cervical prolotherpay for instability with blinded pre and post radiograph reading. Pain Physician 2005;8:321-306. 